nia 19104, USA. Phone: 215.662.9024; Fax: 215.243.1519; E-mail: richard.dunbar@ uphs.upenn.edu.

1. Carlson LA. Nicotinic acid: the broad-spectrum lipid drug. A 50th anniversary review. J Intern Med. 2005;258(2):94-114.

2. National Cholesterol Education Program (NCEP) Expert Panel on Detection, Evaluation, and Treatment of High Blood Cholesterol in Adults (Adult Treatment Panel III). Third Report of the National Cholesterol Education Program (NCEP) Expert Panel on Detection, Evaluation, and Treatment of High Blood Cholesterol in Adults (Adult Treatment Panel III) final report. Circulation. 2002;106(25):3143-3421.

3. Wise A, et al. Molecular identification of high and low affinity receptors for nicotinic acid. J Biol Chem. 2003;278(11):9869-9874.

4. Tunaru S, et al. PUMA-G and HM74 are receptors for nicotinic acid and mediate its anti-lipolytic effect. Nat Med. 2003;9(3):352-355.

5. Soga T, et al. Molecular identification of nicotinic acid receptor. Biochem Biophys Res Commun. 2003;303(1):364-369.

6. Birjmohun RS, Hutten BA, Kastelein JJP, Stroes ESG. Efficacy and safety of high-density lipoprotein cholesterol-increasing compounds: a metaanalysis of randomized controlled trials. J Am Coll Cardiol. 2005;45(2):185-197.

7. Lai E, et al. Effects of a niacin receptor partial ago- nist, MK-0354, on plasma free fatty acids, lipids, and cutaneous flushing in humans. J Clin Lipidol. 2008;2(5):375-383

8. Basketter DA, Wilhelm KP. Studies on non-immune immediate contact reactions in an unselected population. Contact Derm. 1996;35(4):237-240

9. English JS, Winkelmann RK, Louback JB, Greaves MW, MacDonald DM. The cellular inflammatory response in nicotinate skin reactions. BrJ Dermatol. 1987;116(3):341-349.

10. Maciejewski-Lenoir D, Richman JG, Hakak Y, Gaidarov I, Behan DP, Connolly DT. Langerhans cells release prostaglandin D2 in response to nicotinic acid. J Invest Dermatol. 2006;126(12):2637-2646

11. Gille A, Bodor ET, Ahmed K, Offermanns S. Nicotinic acid: pharmacological effects and mechanisms of action. Annu Rev Pharmacol Toxicol. 2008;48:79-106.

12. Benyó Z, Gille A, Bennett CL, Clausen BE, Offermanns S. Nicotinic acid-induced flushing is mediated by activation of epidermal Langerhans cells. Mol Pharmacol. 2006;70(6):1844-1849.

13. Meyers CD, Liu P, Kamanna VS, Kashyap ML. Nicotinic acid induces secretion of prostaglandin D2 in human macrophages: an in vitro model of the niacin flush. Atherosclerosis. 2007;192(2):253-258.

14. Papaliodis D, et al. Niacin-induced "flush" involves release of prostaglandin D2 from mast cells and serotonin from platelets: evidence from human cells in vitro and an animal model. J Pharmacol Exp Ther. 2008;327(3):665-672.

15. Lai E, et al. Suppression of niacin-induced vasodila- tion with an antagonist to prostaglandin D2 receptor subtype 1. Clin Pharmacol Ther. 2007;81(6):849-857.

16. Benyo Z, et al. GPR109A (PUMA-G/HM74A) mediates nicotinic acid-induced flushing. J Clin Invest. 2005;115(12):3634-3640

17. Hanson J, et al. Nicotinic acid- and monomethyl fumarate-induced flushing involves GPR109A expressed by keratinocytes and COX-2-dependent prostanoid formation in mice. J Clin Invest. 2010;120(8):2910-2919.

18. Tang H, Lu JY-L, Zheng X, Yang Y, Reagan JD. The psoriasis drug monomethylfumarate is a potent nicotinic acid receptor agonist. Biochem Biophys Res Commun. 2008;375(4):562-565.

19. Wain EM, Darling MI, Pleass RD, Barker JN, Smith $\mathrm{CH}$. Treatment of severe, recalcitrant, chronic plaque psoriasis with fumaric acid esters: a prospective study. Br J Dermatol. 2010;162(2):427-434

20. Gelfand JM, Neimann AL, Shin DB, Wang X, Margolis DJ, Troxel AB. Risk of myocardial infarction in patients with psoriasis. JAMA. 2006; 296(14):1735-1741

21. Mehta NN, Azfar RS, Shin DB, Neimann AL, Troxel AB, Gelfand JM. Patients with severe psoriasis are at increased risk of cardiovascular mortality: cohort study using the General Practice Research Database. Eur Heart J. 2010;31(8):1000-1006.

22. Grosser T, Fries S, FitzGerald GA. Biological basis for the cardiovascular consequences of COX-2 inhibition: therapeutic challenges and opportunities. J Clin Invest. 2006;116(1):4-15

\title{
PIK3CA and KRAS mutations predict for response to everolimus therapy: now that's RAD001
}

\author{
Morassa Mohseni and Ben Ho Park
}

Breast Cancer Research Program, The Sidney Kimmel Comprehensive Cancer Center at Johns Hopkins, Baltimore, Maryland, USA.

\begin{abstract}
Targeted cancer therapeutics can be effective when patients are preselected to maximize the chance of response. Increasingly, molecular markers such as oncogenic DNA mutations are being exploited to help guide patient preselection. These DNA lesions can predict for either a positive or negative response to a given drug. Finding such predictive biomarkers is an ongoing challenge. New work by Di Nicolantonio and colleagues in this issue of the JCI demonstrates that PI3K catalytic $\alpha$ subunit (PIK3CA) mutations can sensitize cancer cells to the mammalian target of rapamycin (mTOR) inhibitor everolimus. In addition, they show that the concurrent presence of PIK3CA mutations and mutations in either KRAS or BRAF predict for resistance to this drug. These data suggest that mTOR inhibitors currently in use will be ineffective against cancers that have a mutation in either KRAS or BRAF despite having PI3K/AKT/mTOR pathway activation.
\end{abstract}

In the past few decades, developers of new anticancer therapies have moved away from cytotoxic drugs that simply target the proliferative hallmark of all cancer cells. Cur-

Conflict of interest: Ben Ho Park is a consultant for, and has received research funding from, GlaxoSmithKline and is a consultant for Horizon Discovery Ltd.

Citation for this article: J Clin Invest. 2010; 120(8):2655-2658. doi:10.1172/JCI44026. rently, targeted therapies dominate cancer drug development with the aim of blocking the growth and spread of cancer by interfering with specific molecules involved in the progression of a given tumor. One of the most successful targeted anticancer therapies developed is the kinase inhibitor imatinib, which targets the product of the $B C R-A B L$ oncogene that drives disease in all patients with chronic myeloid leuke- mia (CML) (1). However, for most targeted therapies, only a subset of the patients predicted to respond do so. For example, EGFR-directed therapies were thought to inhibit the growth of non-small-cell lung cancers with EGFR overexpression, but only those cancers with certain activating EGFR mutations respond to these small molecule inhibitors $(2,3)$. It has therefore become critically important to develop predictive biomarkers for patients who are likely to respond to a given therapy and, equally important, for those who will not. As an example, testing for KRAS mutations has become mandatory for colorectal cancer patients receiving EGFR-directed therapies because the presence of KRAS mutations predicts for resistance to this class of drugs (4). In this issue of the JCI, Di Nicolantonio and colleagues have now uncovered mutations that seem to predict for response to the anticancer drug everolimus, which targets mammalian target of rapamycin (mTOR) (5). 


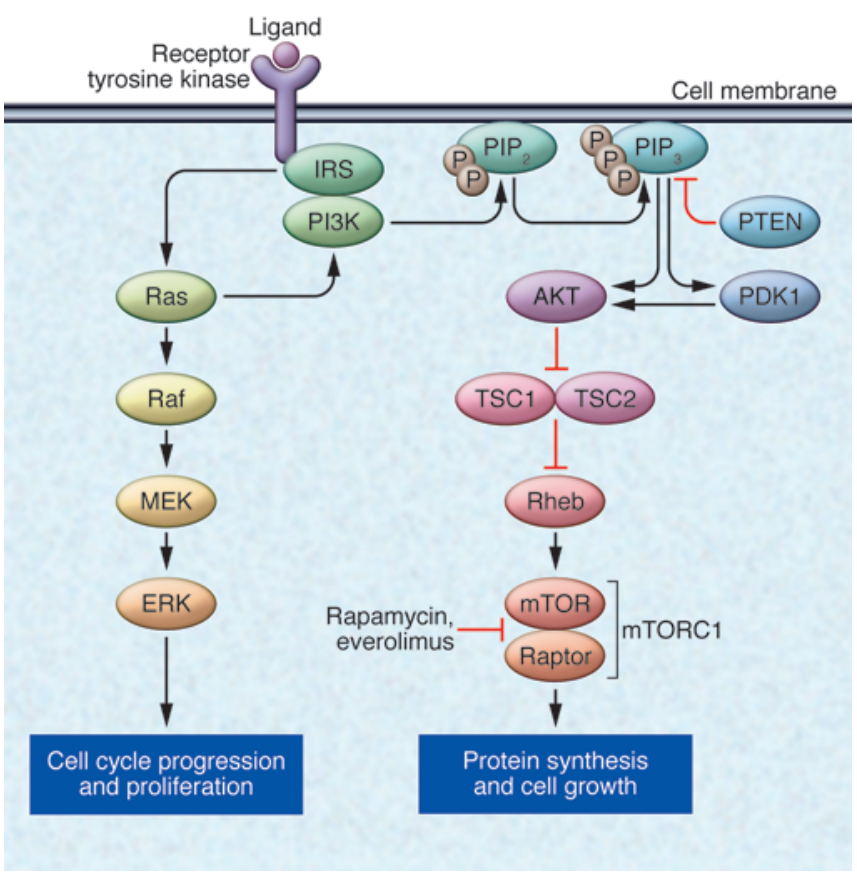

\section{Figure 1}

The PI3K/AKT/mTOR pathway. Receptor tyrosine kinases bind with ligand and initiate the signaling pathway via intermediate molecules (IRS). PI3K becomes activated, which results in phosphorylation of phosphatidylinositol 4,5-bisphosphate $\left(\mathrm{PIP}_{2}\right)$ to phosphatidylinositol 3,4,5trisphosphate $\left(\mathrm{PIP}_{3}\right)$, a process that is reversed by PTEN. At the cell membrane, proteins with pleckstrin homology domains then become phosphorylated via $\mathrm{PIP}_{3}$ (phosphoinositide-dependent protein kinase-1 [PDK1] and AKT). PDK1 can also phosphorylate critical residues on AKT. The tumor suppressor complex of TSC1/TSC2 normally inhibits mTOR activation via Ras homolog enriched in brain (Rheb). Activated AKT prevents this inhibition, leading to activation of the mTOR/Raptor complex known as mTOR complex 1 (mTORC1). This complex can be inhibited by rapamycin and its analogs, including everolimus. Ultimately, mTORC1 leads to the activation of downstream proteins involved in the initiation of protein synthesis, resulting in cellular growth. Receptor tyrosine kinase activation also initiates MAPK pathway signaling, which leads to cell cycle progression and proliferation. MAPK pathway activation can also augment PI3K signaling. MEK, MAPK/ERK kinase.

\section{The PI3K/AKT/mTOR pathway and $\mathrm{mTOR}$ inhibitors}

The PI3K/AKT/mTOR signaling pathway mediates key cellular processes, including cell growth, proliferation, and survival (6) (Figure 1). Activation of the downstream component mTOR can lead to features of transformation through its known role in regulating factors involved with the initiation of protein synthesis of critical growth-promoting genes (7). Furthermore, activating mutations that contribute to carcinogenesis are commonly found in genes encoding proteins within this pathway (8). In particular, oncogenic mutations of PI3K catalytic $\alpha$ subunit (PIK3CA) are among the most frequently reported genetic aberrations in human cancers (9). These mutations activate the PI3K/AKT/mTOR pathway and contribute to carcinogenesis, providing the rationale for inhibiting this pathway for cancer therapy. The development of agents to target components of this pathway has resulted in a class of drugs that specifically target mTOR. However, despite the fact that early-phase clinical trials indicate that mTOR inhibitors may have activity in a number of cancers, only a fraction of patients receiving these drugs derived substantial clinical benefit (10). Developing biomarkers able to stratify patients into those likely to respond and those unlikely to respond is now critical if $\mathrm{mTOR}$ inhibitors are to become widely used for the treatment of cancer.

\section{Cancer mutations and the response to the mTOR inhibitor everolimus}

In this issue of the JCI, Di Nicolantonio and colleagues used a panel of isogenic human cell lines to characterize the response to the mTOR inhibitor everolimus, which is a rapamycin analog originally known as RAD001 (5). This group and others have previously demonstrated that somatic cell gene targeting in non-tumorigenic human cell lines can accurately recapitulate oncogenic mutations and their response to drug therapies $(11,12)$. By using paired cell lines that are isogenic, or as close to isogenic as possible, drug sensitivity versus resistance can accurately be assessed, and any phenotypic changes are the direct result of the introduced mutations (Figure 2). In the current study by Di Nicolantonio and colleagues (5), isogenic human cell lines were generated containing hotspot mutations in PIK3CA (H1047R or E545K) and were found to be selectively sensitive to rapamycin and its analog everolimus. This was true in both spontaneously immortalized non-tumorigenic human breast epithelial cells (MCF10A) and human breast epithelial cells immortalized via telomerase overexpression (hTERT-HME1).

Di Nicolantonio et al. then sought to assess whether the increased sensitivity to everolimus in the non-transformed PIK3CA isogenic human cells could be recapitulated in transformed cancer cells carrying PIK3CA mutations along with multiple other genetic alterations (5). This assessment included screening for everolimus sensitivity using a panel of cancer cell lines with known genetic alterations in PIK3CA or phosphatase and tensin homo$\log (P T E N)$, a tumor suppressor gene that encodes for an inhibitor of the PI3K/AKT/ mTOR signaling pathway. It was in this key experiment that Di Nicolantonio and colleagues discovered that the response to everolimus could be divided into two groups: everolimus-sensitive cancer cells that contained mutations in the PI3K pathway and everolimus-resistant cancer cells that contained mutations in both the PI3K and the MAPK pathways, the latter being characterized as cells with either a KRAS or BRAF mutation.

To test the hypothesis that the presence of a KRAS mutation results in resistance to everolimus, the authors performed additional cell proliferation experiments using cell lines derived from the HCT116 colorectal cancer cell line, which naturally contains a heterozygous PIK3CA mutation as well as a heterozygous KRAS mutation. The team employed HCT116 derivatives that had been previously modified via gene targeting such that the mutant or wildtype KRAS allele had been deleted (13). The cells that contained only a single wild-type 

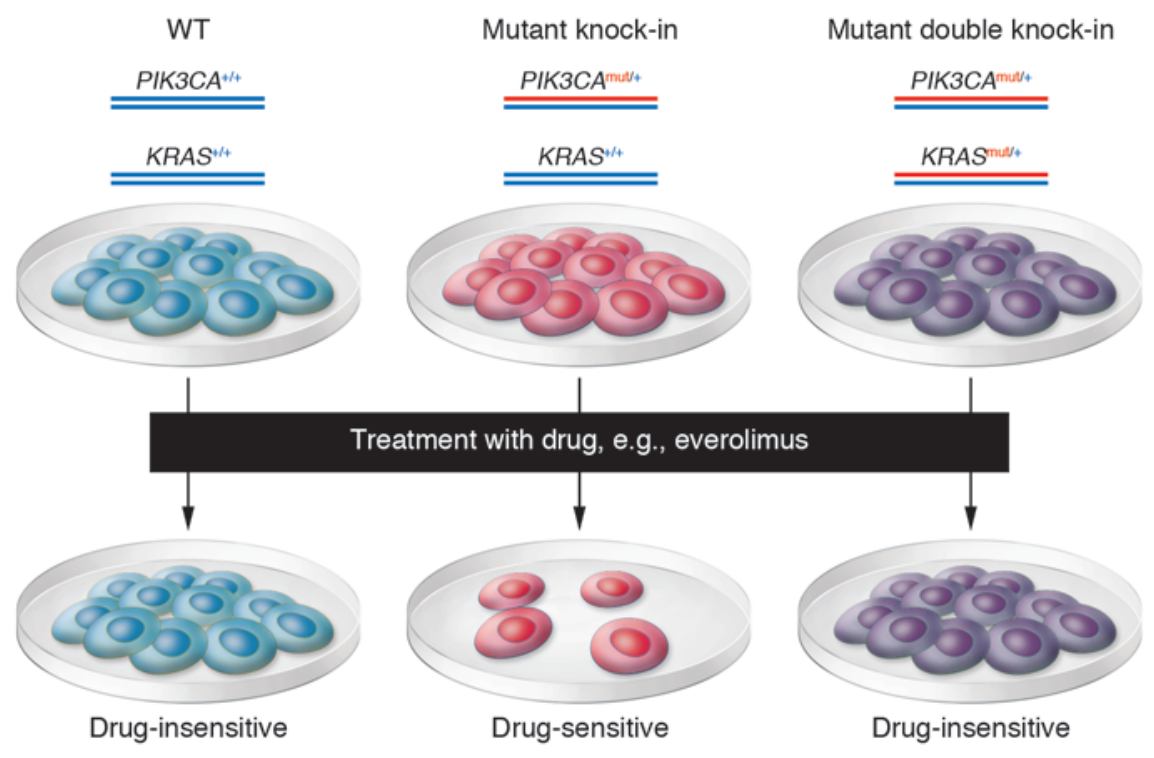

copy of KRAS were sensitive to everolimus, while derivatives of HCT116 containing mutant KRAS were resistant (5). To obtain further evidence that the KRAS mutant was responsible for everolimus resistance, the authors performed rescue experiments on the KRAS wild-type-only HCT116 derivative cell line. By exogenously introducing a mutant copy of KRAS and then treating the cells with everolimus, the authors found that they were able to restore the resistance phenotype.

Di Nicolantonio et al. provide further evidence of the contribution of mutant KRAS in mediating everolimus resistance by assessing whether this finding was relevant in an in vivo setting (5). The authors evaluated this by recapitulating their in vitro data using the above HCT116 system grown as xenografts in immunocompromised mice, as well as a second cell line, ME-180, which is an endometrial cancer cell line that has a PIK3CA mutation but is wild type for KRAS and BRAF. As before, the group generated a derivative of this cell line carrying a transgene overexpressing a mutant KRAS allele. In both mouse xenograft models, the authors found that the presence of mutant PIK3CA as well as a mutant KRAS resulted in abrogation of everolimus's antiproliferative effects.

Importantly, the authors provide data to indicate a potential mechanism by which these KRAS mutations might abrogate the antiproliferative effects of everolimus on cells expressing activating PIK3CA mutations. Using biochemical analyses, they provide evidence that mutant KRAS leads to mTOR-independent protein synthesis, possibly through the activation of $\mathrm{p} 90$ ribosomal S6 kinase (p90RSK).

\section{Clinical implications}

Ultimately, the goal in understanding the mechanism of cancer resistance is to be able to use the information gathered from these laboratory-based experiments and apply them accurately to a clinical setting. Di Nicolantonio and colleagues took their findings and hypothesized that patients with tumors containing both PIK3CA and KRAS mutations would be resistant to everolimus treatment. The authors were able to acquire a small number of tumor samples from patients who had received everolimus therapy and assessed their cancers for PIK3CA mutations and PTEN loss along with BRAF and KRAS mutations. Despite the small number of patients (i.e., 43), the data support the notion that activation of the PI3K pathway by a PIK3CA mutation or PTEN loss does lead to sensitivity to mTOR inhibitors, but that the concurrent presence of either a KRAS or BRAF mutation abrogates this effect. Although it is difficult to know the validity of such analyses given the small sample size, these data strongly support the idea that patients with KRAS or BRAF mutations in their cancers who are receiving everolimus will likely circumvent mTOR inhibition and receive little to no clinical benefit from rapamycin derivatives.

How are we to use the findings of this important study? Like most preclinical data and retrospective studies, the results

\section{Figure 2}

Predicting responses using genetically engineered isogenic human cell lines. Human mammary epithelial cells (WT) are subjected to gene targeting to create isogenic derivatives that contain a single PIK3CA oncogenic mutation (Mutant knock-in) or the same PIK3CA mutation along with a KRAS oncogenic mutation (Mutant double knock-in). Cells are then subjected to drugs in parallel, and resistance versus sensitivity can be assessed. Because the cell lines are isogenic, this allows for a clean interpretation of whether drug sensitivity is mediated by the presence or absence of a given mutation or set of mutations. presented can be viewed as hypothesis generating but not hypothesis proving. Further prospective trials are ultimately needed to validate these results. That said, the results of this elegant study using isogenic cell lines along with mouse models and retrospective patient samples suggest that the new paradigms invoked by the authors may play out to be true in future prospective studies. Moreover, the work of Di Nicolantonio and colleagues (5) presented here provides a framework whereby the use of precise genetic manipulations within human cell lines could be starting points for assessing positive or negative predictors of response to newer targeted therapies. Ultimately, this may lead to more effective identification of patient populations that would be the "right" candidates for a given inhibitor resulting in truly individualized treatments for cancer therapy. The work by Di Nicolantonio et al. is a significant step toward this goal.

\section{Acknowledgments}

M. Mohseni is supported by a Department of Defense Breast Cancer Research Program Predoctoral Award (W81XWH-09-1-0100). B.H. Park acknowledges support from the Breast Cancer Research Foundation, Susan G. Komen for the Cure, the NIH/National Cancer Institute (CA109274, CA88843), and the Avon Foundation.

Address correspondence to: Ben Ho Park, Breast Cancer Research Program, The Sidney Kimmel Comprehensive Cancer Center at Johns Hopkins, 1650 Orleans 
Street, Room 1M42, Baltimore, Maryland 21231, USA. Phone: 410.502.7399; Fax: 410.614.8397; E-mail: bpark2@jhmi.edu.

1. Druker BJ. STI571 (Gleevec) as a paradigm for cancer therapy. Trends Mol Med. 2002;8(4 Suppl):S14-S18.

2. Lynch TJ, et al. Activating mutations in the epidermal growth factor receptor underlying responsiveness of non-small-cell lung cancer to gefitinib. N Engl J Med. 2004;350(21):2129-2139.

3. Paez JG, et al. EGFR mutations in lung cancer: correlation with clinical response to gefitinib therapy. Science. 2004;304(5676):1497-1500.

4. Siena S, Sartore-Bianchi A, Di Nicolantonio F, Balfour J, Bardelli A. Biomarkers predicting clinical outcome of epidermal growth factor receptor-targeted therapy in metastatic colorectal cancer. J Natl Cancer Inst. 2009;101(19):1308-1324.

5. Di Nicolantonio F, et al. Deregulation of the PI3K and KRAS signaling pathways in human cancer cells determines their response to everolimus. J Clin Invest. 2010;120(8):2858-2866.

6. Liu P, Cheng H, Roberts TM, Zhao JJ. Targeting the phosphoinositide 3-kinase pathway in cancer. Nat Rev Drug Discov. 2009;8(8):627-644.

7. Wullschleger S, Loewith R, Hall MN. TOR signaling in growth and metabolism. Cell. 2006; 124(3):471-484

8. Wood LD, et al. The genomic landscapes of human breast and colorectal cancers. Science. 2007; 318(5853):1108-1113.
9. Karakas B, Bachman KE, Park BH. Mutation of the PIK3CA oncogene in human cancers. Br J Cancer. 2006;94(4):455-459.

10. Houghton PJ. Everolimus. Clin Cancer Res. 2010; 16(5):1368-1372

11. Di Nicolantonio F, et al. Replacement of normal with mutant alleles in the genome of normal human cells unveils mutation-specific drug responses. Proc Natl Acad Sci U S A. 2008;105(52):20864-20869.

12. Gustin JP, et al. Knockin of mutant PIK3CA activates multiple oncogenic pathways. Proc Natl Acad Sci U S A. 2009;106(8):2835-2840.

13. Shirasawa S, Furuse M, Yokoyama N, Sasazuki T. Altered growth of human colon cancer cell lines disrupted at activated Ki-ras. Science. 1993; 260(5104):85-88.

\title{
Semaphorin 3E, an exception to the rule
}

\author{
Michael Klagsbrun ${ }^{1,2}$ and Akio Shimizu'
}

1Department of Surgery and 2Department of Pathology, Vascular Biology Program, Children's Hospital Boston, and Harvard Medical School, Boston, Massachusetts, USA.

\begin{abstract}
Class 3 semaphorins (Sema3s) regulate axon guidance, angiogenesis, tumor growth, and tumor metastasis. Neuropilins (NRPs; NRP1 and NRP2) are the cell surface receptors for the Sema3s. However, to signal, interaction of Sema3s and NRPs with plexins is obligatory. In this issue of the JCI, Casazza and colleagues report data that challenge the conventional wisdom about the role of Sema3s in tumor metastasis. As a rule, Sema3B and Sema3F, for example, are inhibitors of tumor angiogenesis, progression, and metastasis. However, Casazza et al. found that Sema3E inhibited tumor growth but atypically promoted invasiveness and metastasis. This metastatic potential was dependent on Plexin D1 expression but was independent of NRP expression. Of clinical importance, Sema3E and Plexin D1 were found to be upregulated in human colon cancer, liver metastasis, and melanoma progression.
\end{abstract}

\section{Semaphorins and their receptors}

There are eight classes of semaphorins characterized by structural heterogeneity $(1,2)$. Class 3 semaphorins (Sema3s; Sema3A-Sema3G) are secreted proteins of approximately $100 \mathrm{kDa}$; they bind neuropilins (NRPs; NRP1 and NRP2) as their cell surface receptors. However, Sema3s also require interactions with plexins to signal. Plexins are large 200-kDa transmembrane proteins that act as substrates for kinases, such as feline sarcoma oncogene (Fes) and Src (3). Plexins form complexes with NRPs to transduce the Sema3 signal. Nine plexins have been identified so far (A1-A4, B1-B3, C1, and D1). Sema3s were originally demonstrated to be axon guidance proteins that repelled axons and collapsed growth cones via NRPs (4). They

Conflict of interest: The authors have declared that no conflict of interest exists.

Citation for this article: J Clin Invest. 2010; 120(8):2658-2660. doi:10.1172/JCI44110. have since been implicated as regulators of angiogenesis and tumor progression $(1,2)$. Sema3A was the first Sema3 to be studied in a vascular context. It was shown to inhibit EC migration and capillary sprouting (5). Subsequent studies showed that Sema3s inhibit adhesion and migration of tumor cells (2). NRPs also bind VEGF family members (6). VEGF-NRP interactions regulate angiogenesis by acting as coreceptors for a receptor tyrosine kinase, VEGFR-2. The puzzle of how two such structurally disparate groups of ligands (Sema3s and VEGF family members) could bind to the same receptor was resolved when it was shown that VEGF binds to the NRP-B domain and that Sema3s bind to the NRP-A domain (7). A critical role for NRPs in angiogenesis, likely as a result of their ability to bind VEGF family members, was shown in mice lacking NRPs (8) and in zebrafish in which NRP levels had been knocked down (9). The convention has been that Sema3s are inhibitors of tumor angiogenesis, progression, and metastasis and that their function requires NRPs. However, in this issue of the JCI, Casazza and colleagues put a new twist on the semaphorin cancer story, particularly in the area of semaphorin effects on metastasis, by demonstrating that Sema3E inhibits tumor growth but promotes metastasis and that it does this in an NRP-independent manner (10).

\section{p61 is the biologically active species} of Sema3E

Sema3E is synthesized as an $85-$ to $90-\mathrm{kDa}$ protein. However, it has furin-sensitive sites that are cleaved to generate p61, which is the main species of endogenous Sema3E. p61 induced lung metastasis in mice (11). In tumor cells, it promoted in vitro cell motility, invasiveness, transendothelial migration, and extravasation. Furin-induced cleavage is a feature of many of the Sema3s (12). For example, Sema3B found in the conditioned medium of cancer cells is almost completely cleaved by furin-like proprotein convertases, generating inactive fragments. So, furin-induced proteolytic processing of Sema3s does not necessarily result in a bioactive form, as it does for Sema3E.

\section{Sema3E binds Plexin D1 but is NRP independent}

Sema3E binds Plexin D1 directly and with high affinity, the only Sema3 to do so (13). A role in angiogenesis for this ligand/ receptor pair has been clearly defined. For 